\title{
16K Array Charge Coupled Device Multi-Ion Image Sensors for Simultaneous Determination of Distributions of Sodium and Potassium Ions
}

\author{
Toshiaki Hattori ${ }^{1,4, *}$, Hikaru Satou ${ }^{1}$, Kenta Tokunaga ${ }^{2}$, \\ Ryo Kato ${ }^{3}$ and Kazuaki Sawada ${ }^{1,4}$
}

\begin{abstract}
${ }^{1}$ Department of Electrical \& Electronic Information Engineering, Toyohashi University of Technology, Hibarigaoka 1-1, Tempaku, Toyohashi, Aichi, 441-8580 Japan ${ }^{2}$ Department of Environmental and Life Science,Toyohashi University of Technology, Hibarigaoka 1-1, Tempaku, Toyohashi, Aichi, 441-8580 Japan ${ }^{3}$ Cooperative Research Facility Center, Toyohashi University of Technology, Hibarigaoka 1-1, Tempaku, Toyohashi, Aichi, 441-8580 Japan ${ }^{4}$ Core Research for Evolutional Science and Technology (CREST), K's Gobancho 6F, 7, Gobancho, Chiyoda-ku, Tokyo, 102-0076 Japan
\end{abstract}

(Received February 18, 2015; accepted September 25, 2015)

Key words: CCD-type multi-ion image sensor, sodium ion, potassium ion, plasticized PVC membrane, inkjet printing

Alkali metal ion sensitive membranes of plasticized poly(vinyl chloride) (PVC) were prepared by an inkjet printer, and a $128 \times 128(16 \mathrm{~K})$ pixel array multi-ion image sensor that simultaneously imaged sodium and potassium ions was developed. The basic sensor chip fabricated from a charge coupled device (CCD) and complimentary metal-oxide semiconductor (CMOS) was used. The sodium-ion-sensitive membrane prepared by an inkjet printer was characterized by comparing the membranes made by conventional casting. The sodium ion membrane made by the inkjet printer had a limited area and thickness, and exhibited a near Nernstian response in 10-20 $\mu \mathrm{m}$ thickness. The multiion image sensor with two kinds of ion-sensitive plasticized PVC membrane was also prepared on the same sensor chip. When $10 \mathrm{mM}$ of sodium ion solution was added into the sample solution of equal volume including $0.1 \mathrm{mM}$ of sodium ions, the region of the sodium-ion-sensitive membrane displayed a large potential change but the region of the potassium-ion-sensitive membrane had no response to the concentration change. When $10 \mathrm{mM}$ of potassium ion solution was added into the sample solution of equal volume including $0.1 \mathrm{mM}$ of potassium ions, the region of the potassium-ion-sensitive membrane displayed a large potential change but the sodium-ion-sensitive membrane did not show a significant change. The $16 \mathrm{~K}$ array CCD multi-ion imaging sensors with a finely striped pattern to monitor sodium and potassium ions functioned successfully.

*Corresponding author: e-mail: thattori@ee.tut.ac.jp 


\section{Introduction}

A plasma membrane has many types of ion channels that control the concentrations of intracellular ions. ${ }^{(1)}$ The dynamic response of cells caused by a certain stimulus indicates specific information about an activity based on biochemical reactions. Ion monitoring of stimulated cells is important to analyze biochemical reactions. The patch clamp is the most useful method to study the dynamics of ion channels, ${ }^{(2)}$ through which $\mathrm{Na}^{+}, \mathrm{K}^{+}, \mathrm{Ca}^{2+}$, and $\mathrm{Cl}^{-}$are transported. A high-density sensor array imager using complimentary metal-oxide semiconductor (CMOS) chips with $16 \mathrm{~K}$ pixels has been used to record neural activity as an alternative to the patch clamp method. ${ }^{(3)}$ Images of the extracellular activity of large-scale neuronal networks have been obtained by a metallic microelectrode array chip with $4 \mathrm{~K}$ pixels. ${ }^{(4)}$ These array sensors allow parallel analysis of many cells without a technically detailed mechanical setup. However, although the arrays monitored the change of a cell's membrane potential, they did not directly monitor the concentration of specific ions.

Array-type ion-sensitive field-effective transistors (ISFETs) with $20 \times 20$ pixels covered with plasticized poly(vinyl chloride) (PVC) membranes have been reported for chemical imaging of several ions and biochemicals. ${ }^{(5,6)}$ The array ISFET sensors required a long time to display a two-dimensional image based on the response of all pixels. We have been developing a real-time charge coupled device (CCD) ion image sensor with a plasticized PVC membrane containing a proper ionophore. The CCD potassium ion image sensor had $32 \times 32(1 \mathrm{~K})$ sensing pixels. It possessed a fine frame of approx. $5 \times$ $5 \mathrm{~mm}^{2}$ and an image speed of 5 frames per second (fps). The $1 \mathrm{~K}$ array CCD potassium ion sensor dynamically monitored potassium ion leakage from a $3.3 \mathrm{M} \mathrm{KCl}$ solution in an $\mathrm{Ag} / \mathrm{AgCl}$ reference electrode. ${ }^{(7)}$ The $1 \mathrm{~K}$ array $\mathrm{CCD}$ sodium ion sensor captured the dynamic images of the ion exchange reaction in a tiny single bead $(\varphi<1 \mathrm{~mm})$ of Na-type cationic exchange resin. It was found that the initial velocity of $\mathrm{Na}^{+}-\mathrm{Ba}^{2+}$ ion exchange was twice as fast as that of $\mathrm{Na}^{+}-\mathrm{Ca}^{2+}$ ion exchange. ${ }^{(8)}$ We further developed CCD ion image sensors with a fast frame rate of $33 \mathrm{fps}$ and a high-density sensing area with 128 $\times 128(16 \mathrm{~K})$ pixels and applied them to dynamic observation in biological systems. The chemical bio-imaging of mast cells as single cells was successful using a $16 \mathrm{~K}$ array CCD organic amine image sensor. ${ }^{(9)}$ A $16 \mathrm{~K}$ array CCD potassium ion image sensor obtained the dynamic images of a living slice of hippocampal tissue stimulated with glutamate. ${ }^{(10)}$ Since the $16 \mathrm{~K} \mathrm{CCD}$ ion image sensor had a wide view area of $5 \times 5 \mathrm{~mm}^{2}$, the setup of small cells can be done easily without stress. Since it had many sensing pixels, moreover, individual data of many cells can do statistical processing only by one experiment with same condition.

As previously reported, ${ }^{(11)}$ we have developed a $1 \mathrm{~K}$ array CCD sodium-potassium multi-ion image sensor. A conventional casting method and an inkjet printing method were tested to prepare two sensing regions on the same sensor. The casting method was fast and easy without special equipment, but it was hard to control each sensing area precisely. The inkjet printer precisely controlled each sensing area. However, the entire region of the sensor chip had to be covered with plasticized PVC because of the ease with which the membrane was delaminated. A partition between the sodium ion sensing area and the potassium ion sensing area was constructed on the $1 \mathrm{~K}$ array CCD multi-ion 
image sensor. The partition was insensitive to either ion and clearly divided the sensing area.

In this study, $16 \mathrm{~K}$ array CCD multi-ion image sensors for simultaneous analysis of sodium and potassium ions with two kinds of plasticized poly(vinyl chloride) membranes were studied. The conditions of the inkjet printing method were examined in detail to improve the ease of delamination, and the partition wall could be removed. The number of coatings by the inkjet printer determined the thickness of the plasticized PVC membrane. The potential responses of the multi-ion image sensor were calibrated with the range of 0.1 to $10 \mathrm{mM}$ for each alkali metal ion. The measured concentration range of sodium ions was lower than the intercellular concentrations of $145 \mathrm{mM}$ in a typical mammal.(12) The concentration range of potassium ions was comparable to the intercellular concentration of $5 \mathrm{mM} .^{(12)}$ The thickness affected the durability of the membrane and the potential response to the primary ion concentration. A two-striped plasticized membrane multi-ion image sensor and alternately painted four-striped multi-ion image sensors were prepared using two different mixed solutions.

\section{Materials and Methods}

\subsection{Reagents}

Bis[(12-crown-4)-methyl]-2-dodecyl-2-methylmalonate [bis(12-crown-4)] and sodium tetrakis[3,5-bis(trifluoromethyl) phenyl]borate (Na-TFPB) were purchased from Dojindo Lab., Inc. (Kumamoto, Japan). PVC (MW = 80000), valinomycin, 1,3,5,7,9,11,13,15-octa(propylmethacryl)pentacyclo[9.5.1.13,9.15,15.17,13] octasiloxane (POSS), and tris(hydroxymethyl)aminomethane (Tris) were purchased from SigmaAldrich, Inc. (St. Louis, MO, USA). Potassium tetrakis (4-chlorophenyl)borate (K-TCPB) was purchased from TCI (Tokyo, Japan). Dioctyl sebacate (DOS), n-dioctyl phthalate (DOP), tetrahydrofuran (THF), cyclohexanone (CHN), cyclohexane, and other reagents were purchased from Wako Pure Chemical Industries, Ltd. (Osaka, Japan). Water deionized by a Milli-Q system was used in all the experiments. Metal ion solutions were prepared daily by dilution from a $1 \mathrm{M}$ stock solution of the metal chlorides.

\subsection{Preparation of plasticized PVC membrane}

Before the preparation of plasticized PVC membranes, a CCD $16 \mathrm{~K} \mathrm{pH}$ ion image sensor ${ }^{(13)}$ was treated with water. The sensor chip was soaked for more than $1 \mathrm{~h}$ in pure water mechanically stirred in a beaker. After the sensor chip was taken out, the water was shaken off. The sensor was dried in a large glove box at room temperature.

The cast solution and printing solution contained the same polymer, plasticizer, ionophre, ion exchanger, and nanofiller. For the sodium-ion-sensitive membrane, PVC of $44.0 \mathrm{mg}$, POSS of $30.0 \mathrm{mg}$, DOP of $22.0 \mathrm{mg}$, Bis(12-crown-4) of $2.7 \mathrm{mg}$, and NaTFPB of $1.3 \mathrm{mg}$ were dissolved in a solvent mixture of $5 \mathrm{~mL}$ THF and $5 \mathrm{~mL} \mathrm{CHN}$. $^{(11)}$ For the potassium ion sensitive membrane, PVC of $44.0 \mathrm{mg}$, POSS of $30.0 \mathrm{mg}$, DOP of $36.0 \mathrm{mg}$, valinomycin of $2.7 \mathrm{mg}$, and K-TCPB of $1.3 \mathrm{mg}$ were dissolved in a solvent mixture of $5 \mathrm{~mL}$ THF and $5 \mathrm{~mL} \mathrm{CHN}$. The above contents were selected in reference to our previous work. ${ }^{(9,11)}$ 
In the cast method, after pouring $20-100 \mu \mathrm{L}$ of the mixed solution onto the sensor chip, the solution was dried for $24 \mathrm{~h}$ at room temperature to form the membrane.

In the inkjet printing method, the printing solution was the same mixed solution. An inkjet apparatus (IJK-200T, Microjet, Shiojiri, Japan) was set up in a large glove box. The nozzle diameter (IJHB-300) was $300 \mu \mathrm{m}$. The apparatus was a piezoelectric dot inkjet printer, and can place the printing solution at the desired point on a chip. A strobe light camera monitored the state of the drops produced. The proper adjustment of two voltage pulses on the operating piezoelectric device formed one drop. The pulse conditions were adjusted daily; $85.0-88.0 \mathrm{~V}$ as the pulse voltages, $25.0-28.0 \mu \mathrm{S}$ as the 1st pulse range, $25.0-28.0 \mu \mathrm{S}$ as the interval range, and $25.0-28.0 \mu \mathrm{S}$ as the 2 nd pulse range. One drop of the cocktail solution covered about $36(6 \times 6)$ sensing pixels. The rate of line printing and the interval of the pitch were $10 \mathrm{~mm} / \mathrm{s}$ and $100 \mu \mathrm{m}$, respectively. The waiting time before the recoating was $10 \mathrm{~s}$. After printing, the membrane was dried for $24 \mathrm{~h}$ at room temperature. Since the inkjet apparatus had only a single injection head, when a multi-ion membrane was prepared, each solution was separately printed.

\subsection{Measurement of ion concentration}

The apparatus for the $16 \mathrm{~K}$ array CCD ion image sensor has been described elsewhere. ${ }^{(9)}$ The sensor possessed 16384 sensing pixels in a two-dimensional $128 \times 128$ array. The dimensions of the sensor area were about $5 \mathrm{~mm}^{2}$. The variable frame speed was constant at 33 frames per second. The sensing region was in a hollow of $\sim 2 \mu \mathrm{m}$ depth, and its size was $13.5 \times 24.5 \mu \mathrm{m}^{2}$. The electrochemical cell consisted of a $\mathrm{Ag} / \mathrm{AgCl}$ electrode/ sample solution/plasticized PVC membrane/semiconductor sensor $\left(\mathrm{Si}_{3} \mathrm{~N}_{4} /\right.$ thin $\left.\mathrm{SiO}_{2} / \mathrm{p}-\mathrm{Si}\right)$. The membrane potential altered the depth of the potential well formed in the $\mathrm{p}$-Si surface under the thin $\mathrm{SiO}_{2}$ layer; the depth of the potential well was measured as the amount of charge. Using a CCD technique, the potential well was filled with electrical charges, and the charge was sent to the output amplifier. Each output signal was sent to an external analog-digital converter and recorded by a computer.

The readout potentials were the value of the potential at the rest time subtracted from the potential at the operation time. The potential slope was evaluated from three different concentrations of sodium ion solutions: $0.1 \mathrm{~mol} / \mathrm{L}(\mathrm{M})$ Tris-buffered $(\mathrm{pH}$ 7.4) solutions with concentrations of 10,1 , and $0.1 \mathrm{mM}$ sodium ions. All signals of the sensing pixels were recorded as a video after each pixel arrayed on the chip was calibrated with the above three solutions. The mean values of the potential slope were calculated from all pixels in the sensor except for bad pixels (dead pixels with no response, poor pixels of less than $30 \mathrm{mV}$, and over-readout pixels of $70 \mathrm{mV}$ ).

\subsection{Measurement of membrane thickness on the chips}

The thickness of plasticized PVC membranes was measured using a laser microscope (Shimadzu SFT-3500, Kyoto, Japan). Since the microscope can barely detect the surface between the membrane and the sensor substrate, the thickness was measured at a border of the membrane. Half the area in a sensor chip was covered with a Kapton film adhesive tape (Teraoka, Tokyo, Japan), and half the membrane was separately prepared under the same conditions previously described. After the tape was peeled off, the thickness 
of the membrane was measured at five points on the border of the peeled region. The five points were positioned in the center of each sensor area that was divided into five sections. The mean value and its relative standard deviation (RSD) were calculated from the five data points.

The thickness of the membrane and its related data (potential slopes and \% of bad pixels of each thickness) were measured for only the sodium ion membrane.

\section{Results and Discussion}

\subsection{Thickness of sodium-ion-sensitive membrane}

Data for the sodium ion membrane thickness on three chips from the cast method are listed in Table 1. The membrane thickness increased as the cast volume of the mixed solution was increased. The increase was linear, and the slope of the increase was $0.140 \mu \mathrm{m} / \mu \mathrm{L}$ with a correlation coefficient of 0.885 . The thickness of five points on the same sensor chip was variable, and the RSD of each chip is large. The deviation in the thickness of five points was not random but strongly biased. The thickness decreased from one side to the other side. In another case, the thickness decreased from the edge to the center. The former may be due to the tilting of the sensor chip during drying. The latter may be due to rapid evaporation of the solvent at the center of the sensor chip.

The thicknesses of the inkjet printing membranes are listed in Table 2. The membrane thickness increased with the number of coatings. The increasing slope was $0.105 \mu \mathrm{m} /$ time with a good correlation coefficient of 0.955 . The RSD for each chip was smaller than that for the cast method. Thus, the inkjet printing method can precisely control thickness of the membrane.

\subsection{Potential response of sodium sensing membrane}

The slopes of the potential responses of the sodium ion image sensor prepared by the casting method and the inkjet method are shown in Fig. 1. The value of the potential slopes is the amount of change of the membrane potential to the common logarithm of the concentration in response to the sodium ion concentration change. The slope of the

Table 1

Membrane thickness by the cast method.

\begin{tabular}{crrrrrrr}
\hline \multirow{2}{*}{$\begin{array}{c}\text { Volume } \\
(\mu \mathrm{L})\end{array}$} & \multicolumn{2}{c}{ Chip 1 } & \multicolumn{2}{c}{ Chip 2 } & \multicolumn{2}{c}{ Chip 3 } & 3 chips \\
\cline { 2 - 7 } & $\begin{array}{c}\text { Mean* } \\
(\mu \mathrm{m})\end{array}$ & $\begin{array}{r}\text { RSD }^{*} \\
(\%)\end{array}$ & $\begin{array}{r}\text { Mean* } \\
(\mu \mathrm{m})\end{array}$ & $\begin{array}{r}\text { RSD* } \\
(\%)\end{array}$ & $\begin{array}{r}\text { Mean* } \\
(\mu \mathrm{m})\end{array}$ & $\begin{array}{r}\text { RSD } \\
(\%)\end{array}$ & $\begin{array}{c}\text { Mean** } \\
(\mu \mathrm{m})\end{array}$ \\
\hline 20 & 4.80 & 9.3 & 4.00 & 13.9 & 2.60 & 27.9 & 3.80 \\
40 & 5.90 & 19.1 & 7.90 & 20.2 & 4.80 & 18.6 & 6.20 \\
60 & 8.20 & 14.5 & 6.80 & 8.6 & 9.00 & 9.6 & 8.00 \\
80 & 11.33 & 20.0 & 10.98 & 17.7 & 13.14 & 23.1 & 11.8 \\
100 & 13.20 & 25.0 & 15.25 & 3.9 & 11.32 & 18.7 & 13.3 \\
\hline
\end{tabular}

"Mean value and RSD of five points.

${ }^{* *}$ Mean value of 3 chips. 
Table 2

Membrane thickness by the inkjet method.

\begin{tabular}{crrrrrrr}
\hline \multirow{2}{*}{$\begin{array}{c}\text { Times } \\
\text { of } \\
\text { coating }\end{array}$} & \multicolumn{2}{c}{ Chip 1 } & \multicolumn{2}{c}{ Chip 2 } & \multicolumn{2}{c}{ Chip 3 } & 3 chips \\
\cline { 2 - 8 } & $\begin{array}{c}\text { Mean } \\
(\mu \mathrm{m})\end{array}$ & $\begin{array}{c}\text { RSD }^{*} \\
(\%)\end{array}$ & $\begin{array}{c}\text { Mean } \\
(\mu \mathrm{m})\end{array}$ & $\begin{array}{c}\text { RSD }^{*} \\
(\%)\end{array}$ & $\begin{array}{c}\text { Mean* } \\
(\mu \mathrm{m})\end{array}$ & $\begin{array}{c}\mathrm{RSD}^{*} \\
(\%)\end{array}$ & $\begin{array}{c}\text { Mean } \\
(\mu \mathrm{m})\end{array}$ \\
\hline 5 & 0.60 & 3.2 & 0.66 & 3.1 & 0.62 & 6.0 & 0.63 \\
10 & 1.02 & 3.2 & 1.43 & 10.0 & 1.11 & 9.9 & 1.19 \\
15 & 1.36 & 10.3 & 1.47 & 4.8 & 1.46 & 1.1 & 1.43 \\
20 & 1.82 & 4.8 & 2.10 & 6.7 & 1.98 & 3.7 & 1.97 \\
30 & 3.18 & 3.0 & 3.58 & 4.5 & 2.64 & 5.6 & 3.13 \\
40 & 4.90 & 6.7 & 4.09 & 7.5 & 3.98 & 13.2 & 4.32 \\
\hline
\end{tabular}

*Mean value and RSD of five points.

${ }^{* *}$ Mean value of 3 chips.

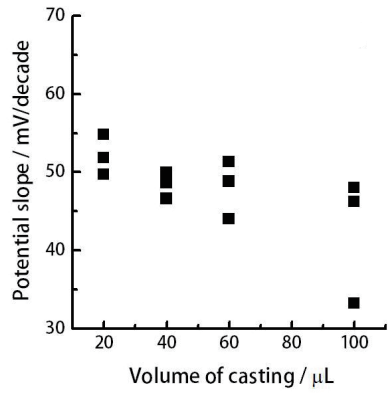

(a)

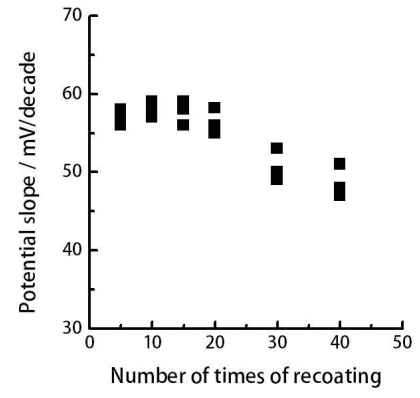

(b)

Fig. 1. Potential response of sodium ion membrane: (a) The casting method and (b) the inkjet printing method.

potential response for the casting method was highly variable in the three chips prepared [Fig. 1(a)]. The slopes indicated less than Nernstian response. The deviation of the potential response for the inkjet method was smaller than for the casting method in the three chips prepared [Fig. 1(b)]. The slopes for 10, 15, and 20 coatings indicated almost Nernstian responses. However, the slope tends to decrease to less than Nernstian response if more than 30 coatings are applied.

\subsection{Durability of plasticized PVC membrane}

When the surface treatment of sensor chips was not carried out, the plasticized PVC membrane sometimes peeled off from the sensor chip during the measurement. The contact between the membrane and the sensor chip was important. Therefore, before inkjet printing, the sensor chip had to be treated so that the printing mixed solution had close contact with the sensor chip. The surface $\left(\mathrm{Si}_{3} \mathrm{~N}_{4}\right)$ of the sensor chip was hydrophobic in the initial state, and it repelled water. The hydrophobic nature of the surface was thought to be useful for the adhesion of organic materials, then, some 
alkyl silane coupling agents were tested to make it more hydrophobic. The alkyl silane treatments, however, were not effective. Next, a few treatments to make the surface hydrophilic were tested; using pure water, dilute hydrochloric acid, or dilute sodium hydroxide. Among them, the most effective treatment was to soak the sensor chip in pure water for $2 \mathrm{~h}$.

The percentages of bad pixels (dead, poor, and over-readout pixels) for the casting method and the inkjet printing method are shown in Fig. 2. The number of bad pixels of the membrane by the casting method was $15-20 \%$. Although the number of bad pixels of the inkjet printing with 5 coatings was more than $25 \%$, that of the other coatings was $8-15 \%$. The membrane with 5 coatings was very thin, so that a part of the membrane had a hole. The good membranes of the inkjet printing may be due to the homogeneity of the membrane contents. Considering the slopes of the potential response and the number of bad pixels, we used 20 coatings to develop the multi-ion image sensor.

\subsection{K array CCD sodium and potassium ion image sensor}

Several snapshots taken of the two-striped multi-ion image sensor are shown in Figs. 3 and 4 . When $100 \mu \mathrm{L}$ of the $10 \mathrm{mM}$ sodium ion solution was added into $100 \mu \mathrm{L}$ of 0.1 $\mathrm{mM}$ sodium ion solution by a $100 \mu \mathrm{L}$ pipette, the color of the upper region immediately changed from blue to red (Fig. 3). The upper region responded to the concentration change of sodium ions, but no color change appeared in the lower region. When 100 $\mu \mathrm{L}$ of the $10 \mathrm{mM}$ potassium ion solution was added into $100 \mu \mathrm{L}$ of $0.1 \mathrm{mM}$ potassium ion solution by a $100 \mu \mathrm{L}$ pipette, the color of the lower region immediately changed from blue to red, but the color of the upper region changed only slightly (Fig. 4). The lower region responded to the concentration change of potassium ions, while the upper region responded only slightly to the concentration change of potassium ions. For a typical mammal the intracellular concentration of sodium ions is 29 times higher than the concentration of potassium ions. ${ }^{(12)}$ Thus, a potassium ion cell-monitoring sensor requires high selectivity. It is very useful in the monitoring of intercellular potassium ions that the present potassium ion sensitive membrane has no significant response to such a high concentration of sodium ions.

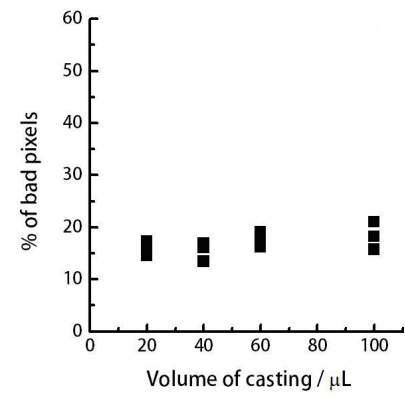

(a)

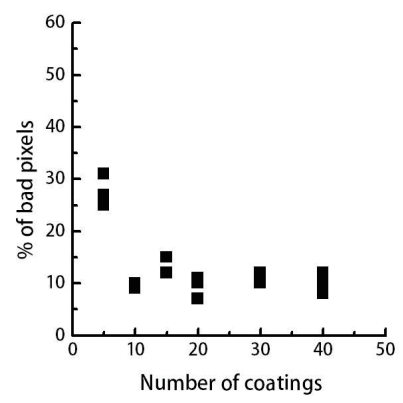

(b)

Fig. 2. The percentage of bad pixels: (a) The casting method and (b) the inkjet printing method. 


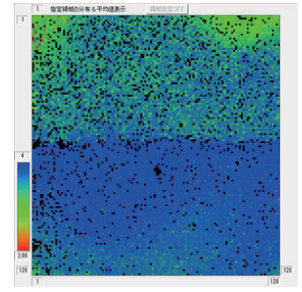

$0 \mathrm{~s}$

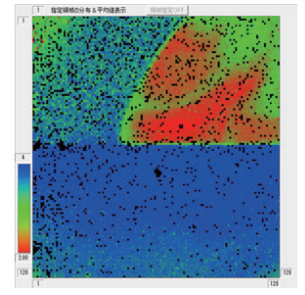

$0.5 \mathrm{~s}$

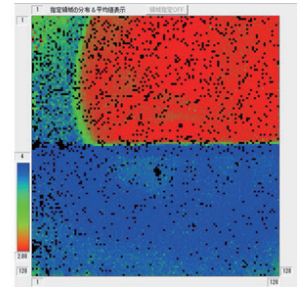

$1.0 \mathrm{~s}$

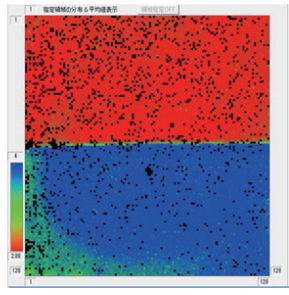

$2.0 \mathrm{~s}$

Fig. 3. (Color online) Snapshots of a two-striped multi-ion image sensor when $10 \mathrm{mM}$ sodium ion solution was injected into a $100 \mu \mathrm{L}$ of sample solution including $0.1 \mathrm{mM}$ sodium ion solution by a $100 \mu \mathrm{L}$ pipette. The upper region and the lower region were a sodium ion sensitive membrane and a potassium ion sensitive membrane, respectively. The concentration profiles display $10 \mathrm{mM}$ (red), $1 \mathrm{mM}$ (green), and $0.1 \mathrm{mM}$ (blue). Each picture has a color scale bar from $10 \mathrm{mM}$ to $0.1 \mathrm{mM}$ at the lower left edge.

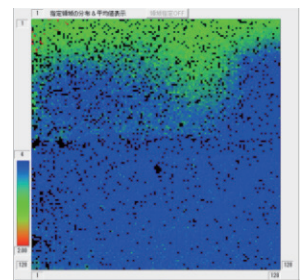

$0 \mathrm{~s}$

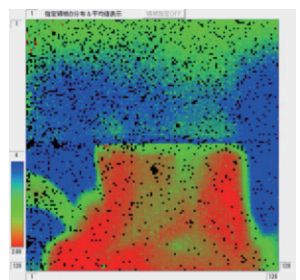

$0.5 \mathrm{~s}$

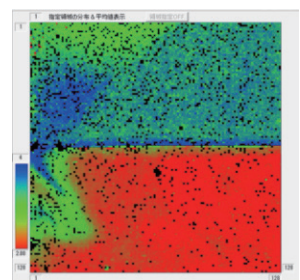

$1.0 \mathrm{~s}$

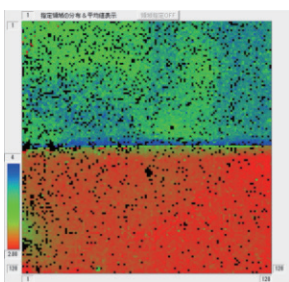

$2.0 \mathrm{~s}$

Fig. 4. (Color online) Snapshots of the same two-striped multi-ion image sensor (Fig. 3) when $10 \mathrm{mM}$ potassium ion solution was injected into a $100 \mu \mathrm{L}$ of sample solution including $0.1 \mathrm{mM}$ potassium ion solution by a $100 \mu \mathrm{L}$ pipette. The concentration color is the same as in Fig. 3. Each picture has a color scale bar from $10 \mathrm{mM}$ to $0.1 \mathrm{mM}$ at the lower left edge.

The time profiles of a pixel in the upper region and a pixel in the lower region are shown in Figs. 5 and 6. Since the added solutions were mixed in the sensor, the time profiles of pixels hollowed with the depth of $\sim 2 \mu \mathrm{m}^{(9)}$ depended on each position. The pixels of Figs. 5 and 6 located just under the point of the injection by the pipette were not strongly affected by the mixture. When the sodium ion solution with high concentration was added (Fig. 5), the sodium ion concentration determined by the upper pixel increased. The potassium ion concentration determined by the lower pixel did not change significantly, except for a transitional signal by fluctuation of the solution just after the addition. When the potassium ion solution of high concentration was added (Fig. 6), the potassium ion concentration increased. The sodium ion concentration also changed slightly in spite of the addition of only potassium ions. This indicated that the sodium-ion-sensitive membrane responded slightly to the change in potassium ion concentration. In intercellular solutions, the concentration of sodium ions is a hundred 


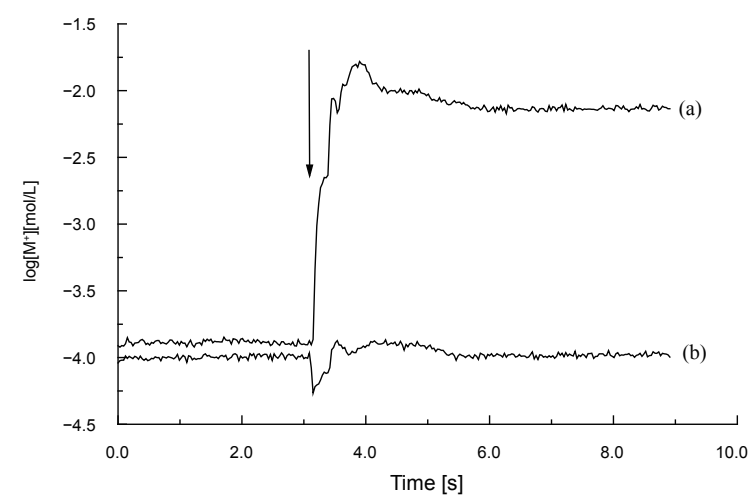

Fig. 5. Time profiles shown by the common logarithm of the concentration in response to the sodium ion concentration change under the same conditions as Fig. 3: (a) a sodium ion sensing pixel and (a) a potassium ion sensing pixel. The arrow indicates the point of addition of the $10 \mathrm{mM}$ sodium ion solution.

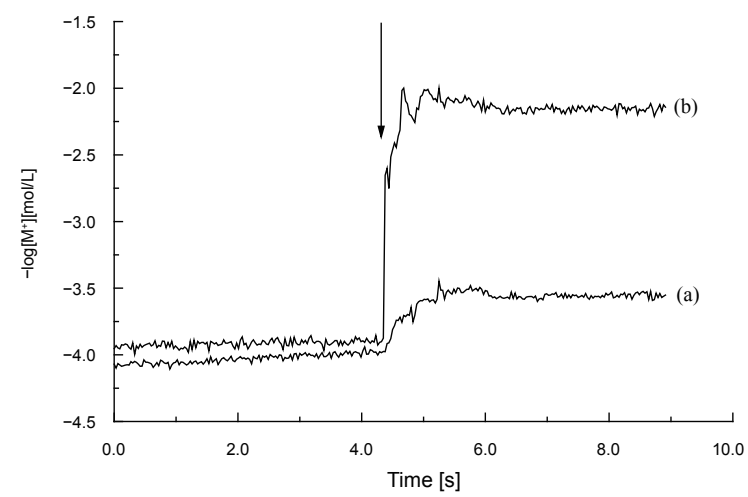

Fig. 6. Time profiles shown by the common logarithm of the concentration in response to the potassium ion concentration change under the same condition as Fig. 4: (a) a sodium ion sensing pixel and (b) a potassium ion sensing pixel. The arrow indicates the point of addition of $10 \mathrm{mM}$ potassium ion solution.

times that of potassium ions. Therefore, the measurement of sodium ions is not affected by potassium ions as such a low concentration. The multi-ion image sensor may monitor the concentration changes of sodium ions and potassium ions individually in intercellular solutions.

The four-striped multi-ion image sensor was also prepared with a sodium-ionsensitive membrane and a potassium ion sensitive membrane. From the upper side of the sensor chip, the first region and the third region were sodium-ion-sensitive membranes. The second region and the fourth region were potassium ion sensitive membranes. 


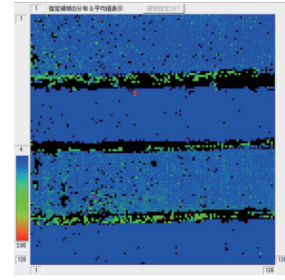

$0 \mathrm{~s}$

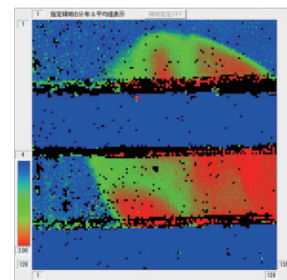

$0.5 \mathrm{~s}$

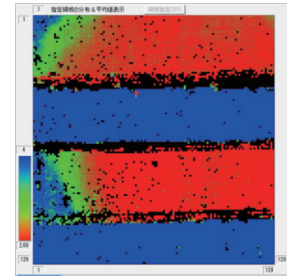

$1.0 \mathrm{~s}$

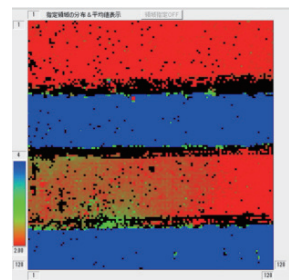

$2.0 \mathrm{~s}$

Fig. 7. (Color online) Snapshots of a four-striped multi-ion image sensor when $10 \mathrm{mM}$ sodium ion solution was injected into a $100 \mu \mathrm{L}$ sample solution including $0.1 \mathrm{mM}$ sodium ion solution by a $100 \mu \mathrm{L}$ pipette. The concentration color is the same as in Fig. 3. Each picture has a color scale bar from $10 \mathrm{mM}$ to $0.1 \mathrm{mM}$ at the lower left edge.

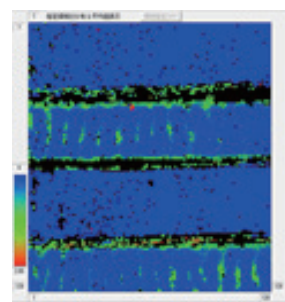

$0 \mathrm{~s}$

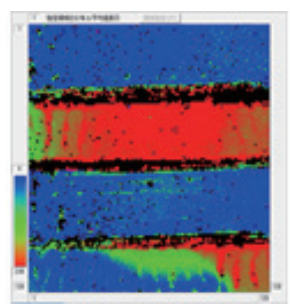

$0.5 \mathrm{~s}$

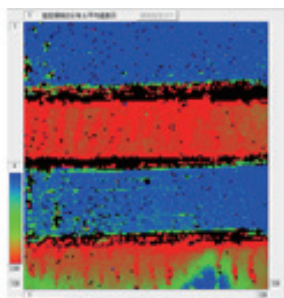

$1.0 \mathrm{~s}$

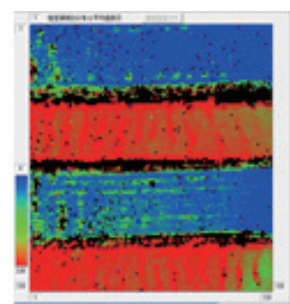

$2.0 \mathrm{~s}$

Fig. 8. (Color online) Snapshots of the same four-striped multi-ion image sensor when $10 \mathrm{mM}$ potassium ion solution was injected into a $100 \mu \mathrm{L}$ sample solution including $0.1 \mathrm{mM}$ potassium ion solution by a $100 \mu \mathrm{L}$ pipette. The concentration color is the same as in Fig. 3. Each picture has a color scale bar from $10 \mathrm{mM}$ to $0.1 \mathrm{mM}$ at the lower left edge.

Several snapshots taken of the four-striped multi-ion image sensor are shown in Figs. 7 and 8. The images in Fig. 7 were taken under the same conditions as Fig. 3. The images in Fig. 8 were taken under the same conditions as Fig. 4. These results of the four-striped multi-ion image sensor also demonstrated a successful simultaneous sensing of sodium and potassium ions.

As previously reported, ${ }^{(9)}$ the vast number of sensing pixels is effective on the statistical processing of biological event to cells. The individual data of many cells can do statistical processing in quite same condition by only one experiment. The multi-ion image sensor would be useful to analyze cell events accompanied by changing sodium ion concentration and/or potassium ion concentration.

\section{Conclusions}

Plasticized PVC membranes for sodium ion sensing and potassium ion sensing were prepared by the inkjet printing method on a $16 \mathrm{~K}$ array CCD ion image sensor chip. The thickness of the membrane was controlled by the number of coatings applied 
by the inkjet printing. The thickness affected the durability of the membrane and the potential response to the primary ion concentration. The multi-ion image sensor with different plasticized PVC membranes was prepared without a partition. The multi-ion image sensor can successfully determine sodium ion concentration and potassium ion concentration simultaneously.

\section{References}

1 B. Hille: Ion Channels of Excitable Membranes, 3rd ed. (Sinauer Associates Inc., Sunderland MA 01375, USA 2001) p. 1.

2 E. Neher, and B. Sakmann: Nature 260 (1976) 799.

3 B. Eversmann, M. Jenkner, F. Hofmann, C. Paulus, R. Brederlow, B. Holzapfl, P. Fromherz, M. Merz, M. Brenner, M. Schreiter, R. Gabl, K. Plehnert, M. Steinhauser, G. Eckstein, D. Schmitt-Landsiedel and R. Thewes: IEEE J. Solid-State Circuits 38 (2003) 2306.

4 L. Berdondini, K. Imfeld, A. Maccione, M. Tedesco, S. Neukom, M. Koudelka-Hep and S. Martinoia: Lab Chip 9 (2009) 2644.

5 H. Meyer, H. Drewer, B. Grundig, K. Cammann, R. Kakerow, Y. Manoli, W. Mokwa and M. Rospert: Anal. Chem. 67 (1995) 1164.

6 H. Meyer, H. Drewer, J. Krause, K. Cammann, R. Kakerow, Y. Manoli, W. Mokwa and M. Rospert: Sens. Actuators B 229 (1994) 18.

7 T. Hattori, Y. Masaki, K. Atsumi, R. Kato and K. Sawada: Anal. Sci. 26 (2010) 1039.

8 T. Hattori, Y. Masaki, D. Mori, R. Miyamoto, R. Kato and K. Sawada: Electroanalysis 24 (2012) 114.

9 T. Hattori, Y. Tamamura, K. Tokunaga, T. Sakurai, R. Kato and K. Sawada: Anal. Chem. 86 (2014) 4196.

10 A. Kono, T. Sakurai, T. Hattori, K. Okumura, I. Makoto and K. Sawada: Sens. Actuator B 201 (2014) 439.

11 T. Hattori, Y. Kojima, K. Tokunaga, Y. Masaki, R. Kato and K. Sawada: Proceedings of The 14th Internatinal Meeting on Chemical Sensors (IMCS 2012) p. 967 (DOI:10.5162/ IMCS2012/P1.3.14).

12 B. Alberts, D. Bray, J. Lewis, M. Raff, K. Roberts and J. D. Watson: Molecular Biology of The Cells, 5th ed. (Garland Science, NY 10016 USA 2015) p. 652.

13 M. Futagawa, D. Suzuki, R. Otake, F. Dasai, M.Ishida and K. Sawada: IEEE Trans. Electron Devices 60 (2013) 2634.

\section{About the Authors}

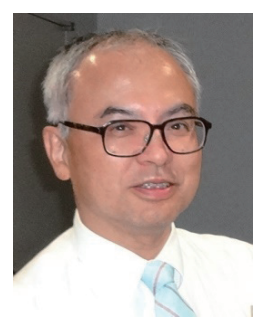

Toshiaki Hattori is an associate professor of the Division of Electrical and Electronic Information Engineering, Toyohashi University of Technology, Japan. He received his Doctor of Science degree from Hokkaido University in 1987. He has studied analytical chemistry including thermometric titration, colloidal titration, electrophoresis, and electrochemical analysis. His current interest focuses on electrochemical bio-image devices for biologically important ions. 


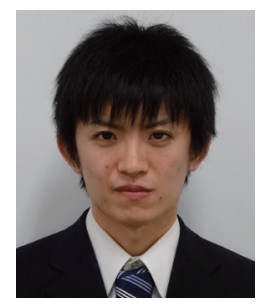

Hikaru Sato received his Bachelor Eng. degree from Toyohashi University of Technology in 2013. He is a master course student of the Division of Electrical and Electronic Information Engineering, Toyohashi University of Technology.

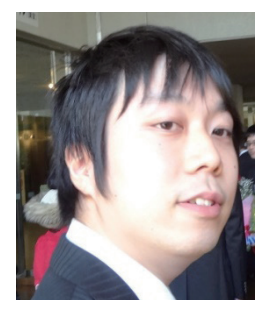

Kenta Tokunaga received his Master Eng. Degree of the Department of Environmental and Life Sciences from Toyohashi University of Technology in 2014. He works in Tokai Chemical Industry Co., Ltd.

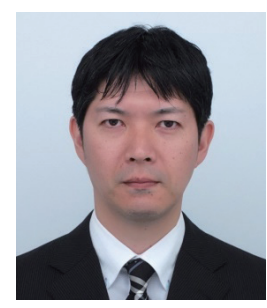

Ryo Kato is an assistant professor of the Cooperative Research Facility Center, Toyohashi University of Technology, Japan. He received his Ph.D. degree in Science from Tohoku University in 2002. His research interests are optical sensing for biologically and environmentally useful anions at liquid/membrane or liquid/solid interfaces.

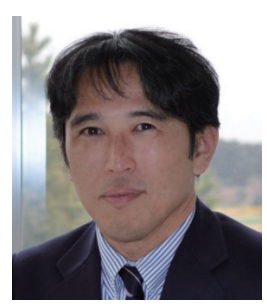

Kazuaki Sawada was born in Kumamoto, Japan in 1963. He received his B.A. and M.S. degrees in electrical and electronic engineering in 1986 and 1988, respectively, and he received a Ph.D. degree in system and information engineering in 1991, all from Toyohashi University of Technology, Japan. From 1991 to 1998, he was a Research Associate in the Research Institute of Electronics, Shizuoka University, Japan. In 1998, he joined the Department of Electrical and Electronic Engineering, Toyohashi University of Technology, where he is now serving as a Full Professor. Professor Sawada is the director of the venture business laboratory, Toyohashi University of Technology. He was a guest professor at the Technical University of Munich in 2005. His current research interests focus on the development of non-label bio-image sensing devices and multimodal sensors for agriculture. 